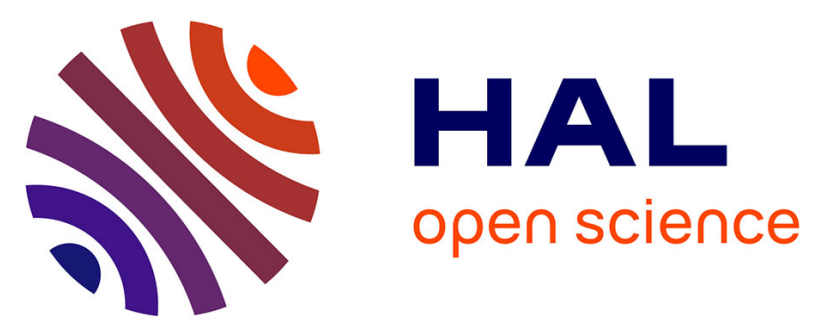

\title{
Toward a High-Stability Coherent Population Trapping Cs Vapor-Cell Atomic Clock Using Autobalanced Ramsey Spectroscopy
}

Moustafa Abdel Hafiz, Grégoire Coget, Michael Petersen, Cyrus Rocher, S Guerandel, Thomas Willette, Emeric de Clercq, Rodolphe Boudot

\section{To cite this version:}

Moustafa Abdel Hafiz, Grégoire Coget, Michael Petersen, Cyrus Rocher, S Guerandel, et al.. Toward a High-Stability Coherent Population Trapping Cs Vapor-Cell Atomic Clock Using Autobalanced Ramsey Spectroscopy. Physical Review Applied, 2018, 9, pp.064002 (1). 10.1103/PhysRevApplied.9.064002 . hal-02387710

\section{HAL Id: hal-02387710 https://hal.science/hal-02387710}

Submitted on 30 Nov 2019

HAL is a multi-disciplinary open access archive for the deposit and dissemination of scientific research documents, whether they are published or not. The documents may come from teaching and research institutions in France or abroad, or from public or private research centers.
L'archive ouverte pluridisciplinaire HAL, est destinée au dépôt et à la diffusion de documents scientifiques de niveau recherche, publiés ou non, émanant des établissements d'enseignement et de recherche français ou étrangers, des laboratoires publics ou privés. 


\title{
Toward a low-drift coherent population trapping Cs cell atomic clock using auto-balanced Ramsey spectroscopy
}

\author{
Moustafa Abdel Hafiz ${ }^{1}$, Grégoire Coget ${ }^{1}$, Michael Petersen ${ }^{1}$, Cyrus Rocher ${ }^{1}$, Stéphane \\ Guérandel $^{2}$, Thomas Zanon-Willette ${ }^{3}$, Emeric de Clercq $^{2}$ and Rodolphe Boudot ${ }^{1}$ \\ ${ }^{1}$ FEMTO-ST, CNRS, UBFC, ENSMM, 26 rue de l'épitaphe 25030 Besançon, France \\ ${ }^{2}$ LNE-SYRTE, Observatoire de Paris, Université PSL, CNRS, \\ Sorbonne Université, 61 avenue de l'Observatoire, 75014 Paris, France \\ ${ }^{3}$ Sorbonne Université, Observatoire de Paris, Université PSL, CNRS, LERMA, F-75005, Paris, France
}

(Dated: March 15, 2018)

\begin{abstract}
Vapor cell atomic clocks are widely appreciated for their excellent short-term fractional frequency stability and their compactness. However, they are known to suffer on medium and long time scales from significant frequency instabilities, generally attributed to light-induced frequency shift effects. In order to tackle this limitation, we investigate the application of the recently proposed auto-balanced Ramsey (ABR) interrogation protocol onto a pulsed hot vapor Cs cell clock based on coherent population trapping $(\mathrm{CPT})$. We demonstrate that the ABR protocol, developed initially to probe the one-photon resonance of quantum optical clocks, can be successfully applied to a two-photon CPT resonance. The applied method, based on the alternation of two successive Ramsey-CPT sequences with unequal free-evolution times and the subsequent management of two interconnected phase and frequency servo loops, is found to allow a relevant reduction of the clock frequency sensitivity to laser power variations. This original ABR-CPT approach, combined with the implementation of advanced electronics laser power stabilization systems, yields the demonstration of a CPT-based Cs cell clock with a short-term fractional frequency stability at the level of $3.1 \times 10^{-13} \tau^{-1 / 2}$, averaging down to the level of $6 \times 10^{-15}$ at $2000 \mathrm{~s}$ integration time. These encouraging performances demonstrate that the use of the ABR interrogation protocol is a promising option towards the development of low drift CPT-based frequency standards. Such clocks could be attractive candidates in numerous applications including next-generation satellite-based navigation systems, secure communications, instrumentation or defense systems.
\end{abstract}

PACS numbers:

\section{INTRODUCTION}

High-performance compact microwave atomic clocks are attractive candidates in numerous applications since they can exhibit modest size, power consumption, cost and excellent fractional frequency stability. Widely-used ubiquitous devices are commercial Cs beam clocks $[1,2]$ and $\mathrm{Rb}$ vapor cell clocks $[3,4]$. These two types of clocks have known over the last decades a large success including their deployment in space applications $[5,6]$. Yet, multiple novel interrogation schemes and techniques are actively pursued and explored towards the development of next-generation high-performance compact vapor cell clocks [7], targeting either reduced dimensions and power or improved frequency stability.

The use of coherent population trapping (CPT) spectroscopy $[8,9]$, allowing to probe a microwave clock transition frequency in an all-optical way, has turned out to be an elegant option for the development of highperformance compact atomic clocks. Vapor-cell CPT clocks have already demonstrated outstanding shortterm instabilities at the level of a few $10^{-13} \tau^{-1 / 2}$ up to $100 \mathrm{~s}$ averaging time [10-13]. However, these clocks suffer in general on longer times scales from significant frequency drifts, preventing them to be fully competitive with best compact vapor cell atomic clocks $[14,15]$. In the following, we will use the expression mid-term for times between 100 and $10^{4} \mathrm{~s}$, and long-term for times greater than $10^{4} \mathrm{~s}$.

A first important contribution to the clock mid-andlong term fractional frequency stability of CPT clocks is the buffer-gas induced temperature-dependent frequency shift, usually measured in fractional value at the level of a few $10^{-10} / \mathrm{K}$ [16-18]. Hopefully, this contribution can be significantly reduced by using a properly-tuned buffer gas mixture [19, 20], stabilizing finely the cell temperature below the $\mathrm{mK}$ level and adopting a well-designed alkali cell geometry to prevent any abnormally large temperature sensitivity [21].

A second major contribution to the mid-and-long-term fractional frequency stability of CPT clocks is known to be light-induced frequency shifts, which depend on the laser power, the laser frequency but also on the CPT sidebands asymmetry through the microwave power of the signal that modulates the laser system [11-13, 20, 22]. These shifts are induced by conventional ac-Stark shifts caused by off-resonant interactions [23] or arise from both resonant optical transitions involved in the $\Lambda$-scheme when incomplete dark states are created [24, 25].

Most of the CPT clocks, including chip-scale atomic clocks [26, 27], operate with the continuous Rabi interrogation scheme. Main drawbacks of CW-regime CPT clocks are to suffer from a significant CPT line power broadening and more especially to exhibit a relevant dependence of the clock frequency to variations of the light field. Typical frequency sensitivities to laser power and 
laser frequency variations in $\mathrm{CW}$-regime $\mathrm{CPT}$ clocks are measured to be in fractional value a few $10^{-12} / \mu \mathrm{W}$ and a few $10^{-12} / \mathrm{MHz}$, respectively $[11,12]$. Several methods have been reported in $\mathrm{CW}$-regime CPT clocks to reduce the sensitivity of the clock frequency to light-field variations. This includes for instance the active stabilization of a specific magic microwave power [22, 28-30], the adjustment of a specific cell temperature [31] or the fine tuning of the laser temperature [28].

An alternative promising option is to use the Ramsey's method of separated oscillating fields [32], in which the single oscillatory field is replaced by a double microwave excitation pulse separated by a free-evolution time. The application of Ramsey spectroscopy with CPT has been reported for the first time on a sodium atomic beam [33] and later investigated in an important number of groups, especially in vapor cell clock experiments [13, 23, 3443]. The Ramsey-CPT method offers the main advantages to produce narrow-linewidth Ramsey-CPT fringes whose linewidth depends mainly on the free-evolution time, and more especially to induce resonant light shifts scaling inversely with the Ramsey period, as shown theoretically and recently experimentally with vapor-cell or cold-atom CPT experiments [13, 44]. This key feature of the Ramsey-CPT method, combined with enhanced phase coherence of the Raman beam and use of an optimized CPT pumping scheme, has allowed recently the demonstration of a low drift cold atom CPT clock exhibiting an Allan deviation of $1.3 \times 10^{-11} \tau^{-1 / 2}$ averaging down to $2 \times 10^{-13}$ after $40000 \mathrm{~s}[45,46]$. At the opposite, the use of Ramsey spectroscopy has not demonstrated so far an improvement in long-term clock stability over continuous interrogation in hot vapor cell CPT clocks $[13,17]$.

Despite its great resolution, the original Ramsey method remains very sensitive to perturbations from the probe field itself. Over the last decade, impressive theoretical efforts have been pursued to propose robust interrogation protocols targeting to eliminate the contribution of the probing-field induced clock frequency shifts. In 2009, A. Taichenachev et al. has proposed for optical clock transitions to extend Ramsey spectroscopy by applying a frequency step to the probing field, equal to the light shift, during the two Ramsey excitation pulses in order to compensate frequency shifts induced by the excitation itself [47]. This method has been improved by the so-called Hyper-Ramsey spectroscopy technique [48], successfully demonstrated onto a $\mathrm{Yb}^{+}$-ion optical clock experiment [49], later extended to the method of modified hyperRamsey (MHR) spectroscopy [50] and to another variant proposed in [51]. In 2016, V. Yudin et al. adapted their synthetic frequency protocol, initially developed to suppress the blackbody radiation shift [52], to an universal interrogation protocol where an error signal is generated from sequences with different dark time intervals and allowing to reduce significantly imperfect correction of probe-induced frequency-shifts even in presence of decoherence due to the laser line-width [53]. This method has been later extended in the general case where both decoherence and relaxation dissipation effects are present [54]. In this case, the error signal is calculated from the combination of several error signals generated from different generalized Hyper-Ramsey spectroscopy sequences. More recently, a novel interrogation protocol named auto-balanced Ramsey (ABR) spectroscopy has been proposed [55]. This method is based on the combination of two successive Ramsey sequences with unequal free evolution times, yielding the management of two interconnected phase and frequency servo loops. The first Ramsey cycle, with short free-evolution time, allows to extract an error signal used to apply a phase step correction to the interrogation field between the Ramsey pulses. The second Ramsey cycle, with long free-evolution time, generates an error signal used to lock the probing field mean frequency. The efficiency of this method has been experimentally demonstrated in an ${ }^{171} \mathrm{Yb}^{+}$ion optical clock, yielding a final reduction by about $10^{4}$ of the light shift coefficient [55].

In this article, we report the in-progress frequency stability performance improvement of a CPT-based Cs cell atomic clock. Advanced electronics dedicated to stabilize the laser power in the pulsed regime and improved thermal isolation of the experiment have been first implemented, yielding a clock fractional frequency stability at the level of $1.4 \times 10^{-13} \tau^{-1 / 2}$ up to $200 \mathrm{~s}$ and $1 \times 10^{-13}$ at $10^{4} \mathrm{~s}$. More interestingly, we demonstrate here theoretically and experimentally the possible application of the ABR-interrogation protocol to a CPT-based Cs cell atomic clock. Compared to the usual RamseyCPT regime, the ABR-CPT protocol is found to allow a relevant reduction of the clock frequency dependence to laser power variations, yielding at $2000 \mathrm{~s}$ averaging time an improvement by a factor 6 of the clock Allan deviation measured to be $6 \times 10^{-15}$. These performances at 2000 $\mathrm{s}$ are a factor 33 better than our previously published results [13].

\section{A SIMPLE MODEL FOR AUTO-BALANCED RAMSEY WITH CPT}

We present here a simple model based on materials from refs $[56,57]$ in order to support the experimental demonstration reported in section $\mathrm{V}$ of enhanced immunity to probing field-induced frequency-shifts using the auto-balanced Ramsey-CPT (ABR-CPT) interrogation protocol. We suppose a basic three-level system excited by two coherent lasers where the first pulse is long enough to allow a complete atomic preparation into the dark state superposition and a second delayed very short pulse to probe that superposition. The three-level system includes two ground-state hyperfine levels, labeled 1 and 2, and an excited level noted 3. The optical transitions are driven by Rabi frequencies $\Omega_{1,2}$, where subscripts 1,2 refer to the involved ground state. The spontaneous emission rates $\Gamma_{31}, \Gamma_{32}$, optical coherence relaxations $\gamma_{1,2}$ and 


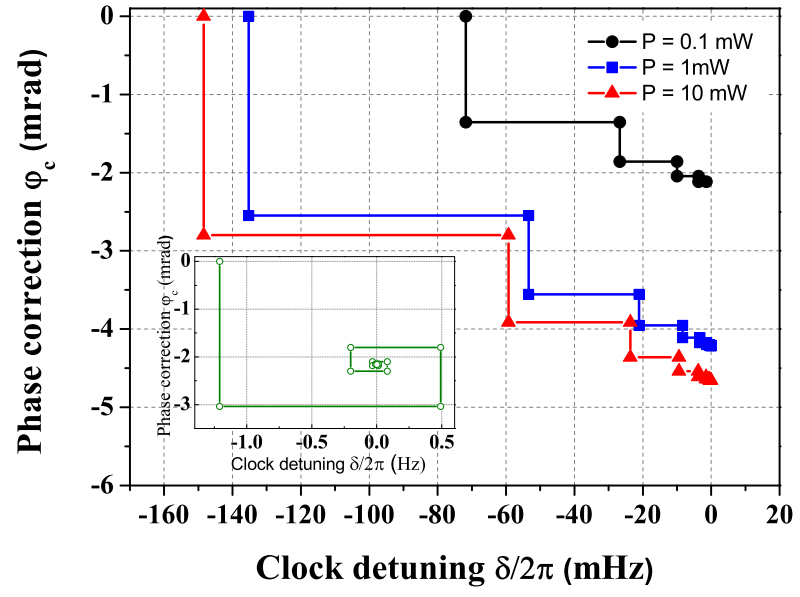

FIG. 1: Trajectory in the phase space after closing both servoloops. $\mathrm{T}_{1}=5 \mathrm{~ms}, \mathrm{~T}_{1} / \mathrm{T}_{2}=2.5$. Black dots: $\mathrm{P}=0.1 \mathrm{~mW}$, Blue squares: $\mathrm{P}=1 \mathrm{~mW}$, red triangles: $\mathrm{P}=10 \mathrm{~mW}$. Inset: particular case, $\mathrm{T}_{1}=0.5 \mathrm{~ms}, \mathrm{~T}_{1} / \mathrm{T}_{2}=5, \mathrm{P}=0.1 \mathrm{~mW}$.

a Raman decoherence $\gamma_{c}$ are taken into account. We suppose in the adiabatic regime that the short probe pulse operation is well described by a stationary line-shape with Raman-Ramsey CPT fringes including a phase-shift expression free from any time dependence as in [56].

For a free evolution time $T$, the oscillating part of the Raman-Ramsey signal can be written as $\cos (\delta T-\Phi)$, with $\delta$ the Raman detuning (i.e. laser frequency difference minus ground-state hyperfine splitting), and $\Phi$ the accumulated effective phase including the light-induced frequency shifts during the optical pulses. In order to stabilize the frequency of a local oscillator (LO) on the hyperfine splitting frequency, a phase modulation $\pm \pi / 2$ is applied between two sequential pulses. A dispersionshape error signal is then obtained, scaling as $\sin (\delta T-\Phi)$, by differentiating two successive Ramsey signals. In usual Ramsey experiments, the probe frequency is locked with a detuning $\delta=\Phi / T$. The originality of the ABR technique [55] is the use of two alternate Ramsey sequences, one with a long free evolution time $\mathrm{T}_{1}$, the other with a shorter time $\mathrm{T}_{2}$, and two interleaved servo-loops. The error signal generated by the $\mathrm{T}_{1}$ sequence is then used to lock the frequency at $\delta=0$, while the error signal of the $\mathrm{T}_{2}$ sequence allows to correct $\Phi$ with an additional phase step $\varphi_{c}$, such that $-\Phi+\varphi_{c}=0$.

To demonstrate analytically the robustness of a phasefrequency stabilization on the unperturbed Raman clock transition by auto-balanced Ramsey spectroscopy, we give the explicit form of the set of interleaved loop equations, expressed as follows:

$$
\left.\delta\left(\varphi_{c}\right) \hookrightarrow \begin{array}{l}
\delta \mathrm{T}_{1}-\Phi(\delta)+\varphi_{c}=0 \\
\delta \mathrm{T}_{2}-\Phi(\delta)+\varphi_{c}=0
\end{array}\right) \varphi_{c}(\delta),
$$

where $\varphi_{c}$ is applied before the second Ramsey-CPT pulse of each sequence. In our simplified model the probe- induced shifts $\eta_{1}, \eta_{2}$ are introduced in the optical detunings as follows $\Delta_{1}=\eta_{1}+\delta / 2$ and $\Delta_{2}=\eta_{2}-\delta / 2$, where we assume $\eta_{1}, \eta_{2}$ proportional to $\Omega_{1}^{2}, \Omega_{2}^{2}$, respectively. The phase-detuning relationship for an ABR-CPT interrogation scheme eliminating probe-induced shifts is given by a simple approximated expression for the Raman phase $\Phi(\delta)$ when $\Omega_{1,2} \ll \Gamma$ as $[56]$ :

$$
\Phi(\delta) \approx \operatorname{Arctan}\left[\frac{\operatorname{Im}\left\{\rho_{12}\right\}}{\operatorname{Re}\left\{\rho_{12}\right\}}\right]=\frac{\left(\Delta_{1}-\Delta_{2}\right)+\bar{\Delta} \gamma_{c}}{\left(\Delta_{1}-\Delta_{2}\right) \bar{\Delta}-\gamma_{e f f}},
$$

where $\rho_{12}$ is the hyperfine coherence in the density matrix formalism. Other parameters $\gamma_{\text {eff }}$ and $\bar{\Delta}$ are defined as:

$$
\gamma_{e f f}=\gamma_{c}+\frac{\Omega_{1}^{2}}{\gamma_{2}}+\frac{\Omega_{2}^{2}}{\gamma_{1}}
$$

and

$$
\bar{\Delta}=\frac{\Omega_{2}^{2} \Gamma_{31} \gamma_{2} \Delta_{1}-\Omega_{1}^{2} \Gamma_{32} \gamma_{1} \Delta_{2}}{\gamma_{1} \gamma_{2}\left(\Omega_{2}^{2} \Gamma_{31}+\Omega_{1}^{2} \Gamma_{32}\right)} .
$$

The set of equations given by Eq. (1) are automatically converging after an iterative process to the set of following stable parameters:

$$
\delta\left(\varphi_{c}\right)=0, \quad \varphi_{c}=\left.\Phi\left(\eta_{1}, \eta_{2}\right)\right|_{\delta=0} .
$$

An example of the system trajectory in the phase space $\left(\delta, \varphi_{c}\right)$ after closing both loops is shown in Fig. 1 for three laser powers and a ratio $\mathrm{T}_{1} / \mathrm{T}_{2}=2.5$. The starting points are different because the probe induced shifts are different. A detuning $\delta=0$, i. e. without frequency shift, is reached in only a few steps, demonstrating the method efficiency. It can be noted that in particular cases the trajectory can be different of a staircase shape, as seen in the inset of Fig. 1 where a spiral shape is observed for $\mathrm{T}_{1} / \mathrm{T}_{2}=5$ and $\mathrm{T}_{1}=0.5 \mathrm{~ms}$. For this study, we used approximated experimental parameter values extracted from [13], including broadening effects on optical lines by buffer gas as following:

$$
\begin{array}{r}
\Gamma_{31}=\Gamma_{32} \sim \Gamma / 2=2 \pi \times 200 \mathrm{MHz}, \\
\gamma_{1}=\gamma_{2}=\left(\Gamma_{31}+\Gamma_{32}\right) / 2 \sim \Gamma / 2, \\
\gamma_{c} \sim 2 \pi \times 400 \mathrm{~Hz}, \\
\eta_{1}-\eta_{2} \sim 2 \pi \times 7.5 \times 10^{-12} \Omega^{2} \mathrm{~Hz}, \\
\mathrm{~T}_{1} \sim 5 \mathrm{~ms}, \quad \mathrm{~T}_{2} \sim 2 \mathrm{~ms}, \\
\Omega_{1}=\Omega_{2} \sim 2.10^{6} \times \sqrt{P / S},
\end{array}
$$

where $P$ is the light power and $S=3 \times 10^{-4} \mathrm{~m}^{2}$ is the beam area.

\section{EXPERIMENTAL SETUP}

Figure 2 describes the CPT-based Cs cell clock schematic. The latter, previously described in [13], combines an optimized CPT pumping scheme named pushpull optical pumping (PPOP) $[58,59]$ and a pulsed interrogation scheme. The laser source is a distributed 


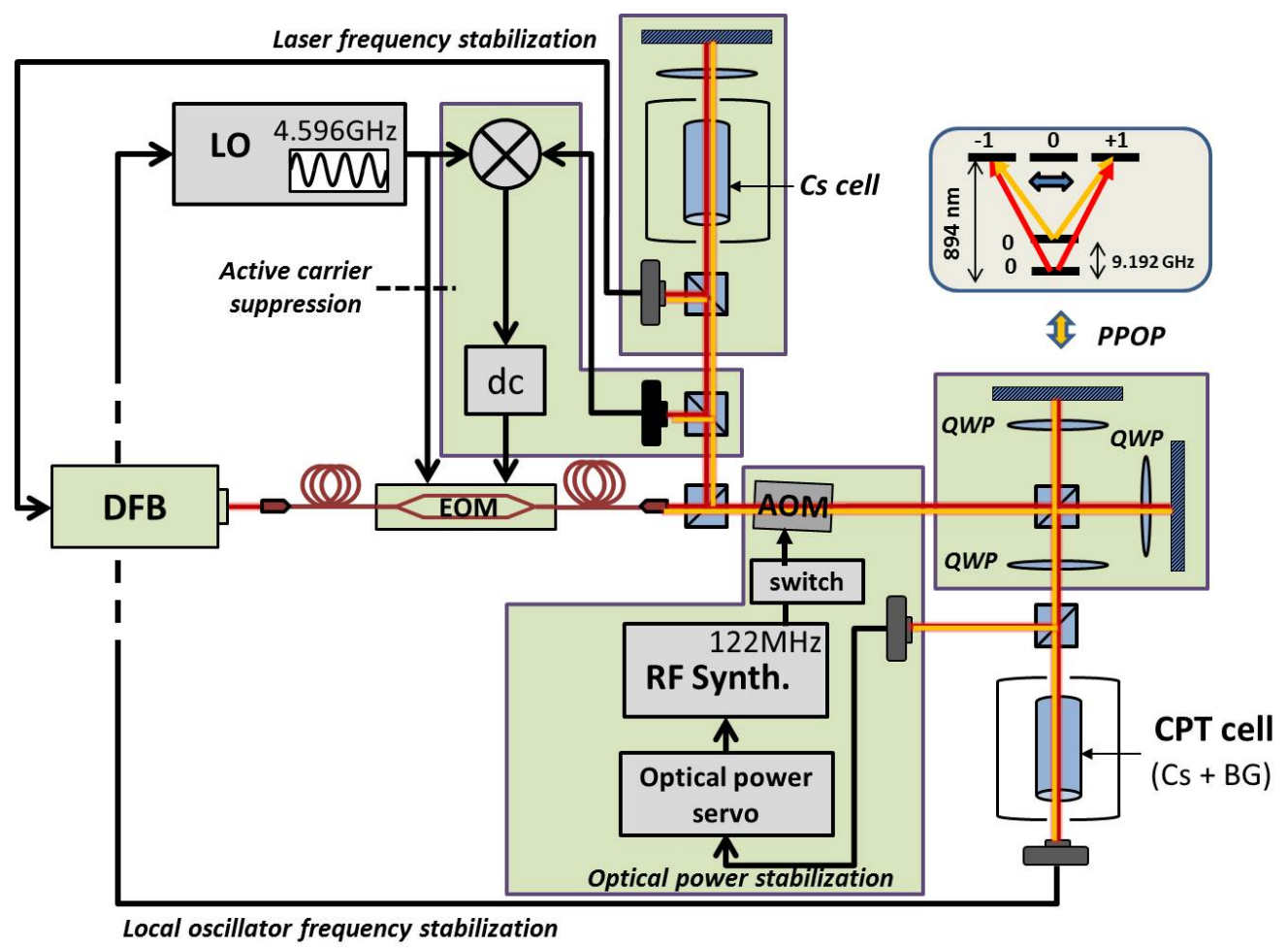

FIG. 2: Schematic of the CPT-based clock. DFB: distributed feedback laser, EOM: Mach-Zehnder electro-optic modulator, AOM: acousto-optic modulator, RF synth.: radio-frequency synthesizer used to drive the AOM, switch: microwave switch used to produce the pulsed light sequence, LO: local oscillator, dc: dc bias voltage applied onto the MZ EOM, Cs+BG: buffer gas-filled Cs vapor cell. The total optical path length difference between both arms of the Michelson system is $\lambda_{C s} / 2 \sim 16.2$ $\mathrm{mm}$ where $\lambda_{C s}$ is the clock transition wavelength. The full clock operation, including the control of the $4.6 \mathrm{GHz}$ synthesizer (phase and frequency), is ensured by a multi-function card (NI-USB6259) and a computer (not shown). The inset shows the push-pull optical pumping (PPOP) CPT-scheme diagram involved in the experiment.

feedback (DFB) diode laser tuned on the Cs $\mathrm{D}_{1}$ line at $894.6 \mathrm{~nm}$. A 70-dB optical isolation stage is implemented at the output of the laser to prevent optical feedback. The laser light is injected into a temperature-stabilized fibered Mach-Zehnder intensity electro-optic modulator (MZ EOM). The latter is modulated at $4.596 \mathrm{GHz}$ by a low phase noise microwave frequency synthesizer [60] in order to produce two highly-coherent first-order optical sidebands frequency-split by $9.192 \mathrm{GHz}$. At the output of the EOM, a dual-frequency sub-Doppler spectroscopy system in a pure Cs reference cell is implemented for stabilization of the laser frequency $[61,62]$ onto the Cs $F^{\prime}=4$ excited-state. The other fraction of the light is sent into an acousto-optic modulator (AOM) driven by a radio-frequency $(\mathrm{RF})$ synthesizer in order to shift the laser frequency by $-122 \mathrm{MHz}$ and compensate for the buffer gas optical frequency shift in the CPT cell [63]. The signal from the AOM RF synthesizer driver can be switched on and off in order to produce the pulsed CPT interaction. The laser beam is then sent into a Michelson-like system allowing to produce the PPOP scheme $[58,59]$, in which atoms interact with two bichromatic optical fields, exhibiting orthogonal circular polarizations and a half-clock period delay. This pump- ing scheme helps to generate the constructive interference of two successive dark states, yielding most of the atoms whatever their initial state to be pumped into the $0-0$ clock transition. At the output of the Michelson system, a beam splitter is used to divide the laser beam into two sub-beams. The first sub-beam is detected by a low noise photodiode associated to a home-made optical power servo system operating in the pulsed regime (see section IV). The other sub-beam, expanded and collimated to a diameter of $2 \mathrm{~cm}$, is sent towards a Cs vapor cell (2-cm diameter and 5-cm length) filled with a buffer gas mixture of nitrogen and argon (pressure ratio $\left.r=P_{A r} / P_{N_{2}}=0.6\right)$ of total pressure 15.3 Torr. The cell is temperature-stabilized at $35^{\circ} \mathrm{C}$. A solenoid is used to generate a static magnetic field of $5 \mu \mathrm{T}$ parallel to the laser beam, in order to make arise the Zeeman manifold. The cell ensemble is protected by a double-layer mu-metal magnetic shield. The laser beam at the output of the cell is focused using a converging lens and detected by a photodiode.

The full clock operation is ensured using a multifunction data acquisition card piloted by a computer and a dedicated Python software. Figure 3 describes the RamseyCPT sequences performed in the usual Ramsey-CPT and 
ABR-CPT regimes, respectively.

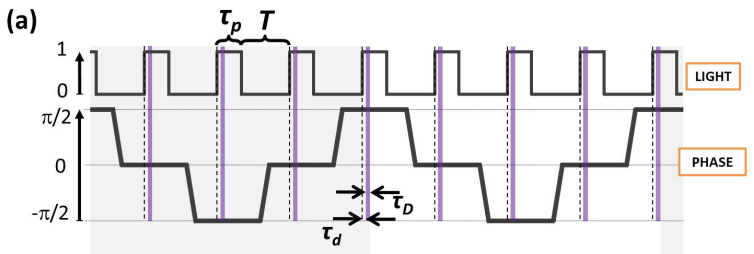

(b)

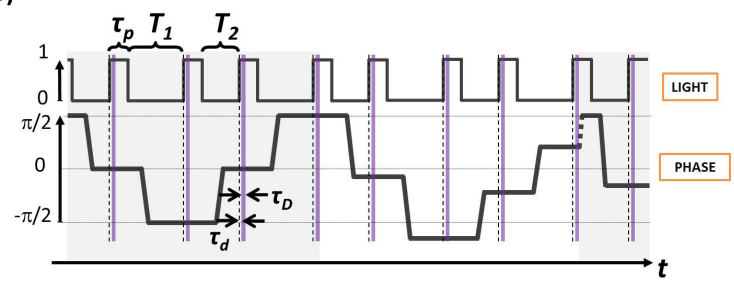

FIG. 3: Pulsed CPT sequence. (a): Ramsey-CPT (with phase modulation), (b): ABR-CPT. The atoms interact with the light during pulses of length $\tau_{p}$, spaced apart by free evolution times $T, T_{1}$ or $T_{2}$. Each pulse is the second pulse of the previous Ramsey interrogation and the first one of the next Ramsey interrogation. The signal is detected at the beginning of each pulse, after a delay time $\tau_{d}$, and averaged during $\tau_{D}$. The vertical dashed lines show the rising edges of the pulses, the solid vertical lines show the detection time. For more clarity, in (b), the phase sequence is shown without $\varphi_{c}$ correction in the first shaded area, and with correction outside.

In the Ramsey-CPT case, atoms interact with regular optical CPT pulses. The first CPT pulse pumps the atoms into the dark state using a typical duration $\tau_{p}$ of $1.1 \mathrm{~ms}$. Atoms evolve then freely in the dark during a free-evolution time $T$ of $2.7 \mathrm{~ms}$ before being detected at the next pulse, after a delay time $\tau_{d}$ of $20 \mu$ s during a detection window of duration $\tau_{D}$ of $80 \mu \mathrm{s}$. The clock signal is averaged over the detection window with a rate of 1 Msamples/s. The sequence cycle duration is noted $T_{c}=\tau_{p}+T$. The associated cycle frequency is noted $f_{c}=1 / T_{c}$. The output mean phase of the local oscillator (LO) is modulated at the frequency $f_{m}=1 /\left(4 T_{c}\right)$. The phase is modified during the dark time. Smooth $50 \mu \mathrm{s}$ ramp-based variations of the $\mathrm{LO}$ output phase are applied instead of pure square-wave phase modulation in order to avoid any LO phase transient perturbations due to the LO phase modulation. A $-\pi / 2$-step is applied each first and second cycle of a period, and a $\pi / 2$-step is applied each third and fourth cycle. The averaged CPT signal is synchronously demodulated in order to produce an error signal that zeroes when the LO frequency matches the $\mathrm{CPT}$ resonance central frequency. A correction is applied to the LO frequency at the end of each period, just after the last detection window.

In the ABR-CPT method (see Fig. 3(b)), the sequence is based on the alternation of two successive Ramsey-CPT cycles with unequal free evolution times $T_{2}=2.7 \mathrm{~ms}$ and $T_{1}=5.4 \mathrm{~ms}$. The detection section is analog to the standard Ramsey-CPT case. The phase is twice decreased by $\pi / 2$ in the middle of the first short and long dark times and increased twice again by $\pi / 2$ in the middle of the following short and long dark times. An additional phase jump $\varphi_{c}$ is applied at each $\pi / 2$ phase transition. For each iteration, two error signals, one for dark times $T_{2}$ and $T_{1}$, are computed. The error signal extracted from the cycle with the short dark time $T_{2}$ is used to compute the value of the phase jump $\varphi_{c}$ applied in the next iteration. The error signal extracted from the cycle with the long dark time $T_{1}$ is used to correct the LO frequency during the next iteration. As the mean phase of the $4.596 \mathrm{GHz}$ synthesizer (see [60]) is incremented of $\varphi_{c} / 2$ (i.e. $\varphi_{c}$ on the 9 $\mathrm{GHz}$ signal) using a DDS after each Ramsey-CPT pulse, the average DDS phase modulation control voltage varies monotonically along time and eventually reaches its extreme value. In order to circumvent this issue and since only the phase step between two pulses is significant, the mean phase of the DDS is reset to zero (as sketched in Fig. 3 by the bold dashed line) immediately after the end of the last detection window of each ABR total period in order to compensate for the absolute phase drift. In both Ramsey-CPT and ABR-CPT cases, the clock output signal is counted by comparison with the signal of a reference active hydrogen maser [64].

\section{OPTICAL POWER STABILIZATION AND THERMAL ISOLATION}

In a first approach, enhanced thermal isolation of the experiment and laser power stabilization have been implemented in order to improve the clock frequency stability performances.

The temperature fluctuations of the optical table are reduced using two passive polyurethane foam-based insulation boxes. The first box, being priorly the unique box to be used, covers the total top surface of the optical table. The second box, made of 10-cm thick polyurethane foam, has been added in order to surround the whole experimental setup, including the first isolation box. The bottom surface of the second box is placed between the optical table breadboard bottom surface and the optical table legs in order to improve the setup thermal isolation from thermal fluxes coming from the ground and table legs. Figure 4 reports the typical temperature fluctuations of the laboratory room and of the experimental setup in respective cases where only the first box is used (past configuration) or where both boxes are implemented.

The laboratory room temperature fluctuations are measured to be a few tens of $\mathrm{mK}$ for time scales up to $10000 \mathrm{~s}$. With the first isolation box, temperature fluctuations are reduced by a factor 2.6 and 6 at $5 \mathrm{~s}$ and $1000 \mathrm{~s}$ averaging time, respectively. The addition of the second isolation box allows to reduce further the temperature fluctuations by a factor 7.9 and 12 at $5 \mathrm{~s}$ and $100 \mathrm{~s}$ averaging time, respectively. For time scales higher than about $1000 \mathrm{~s}$, the thermal isolation of the 


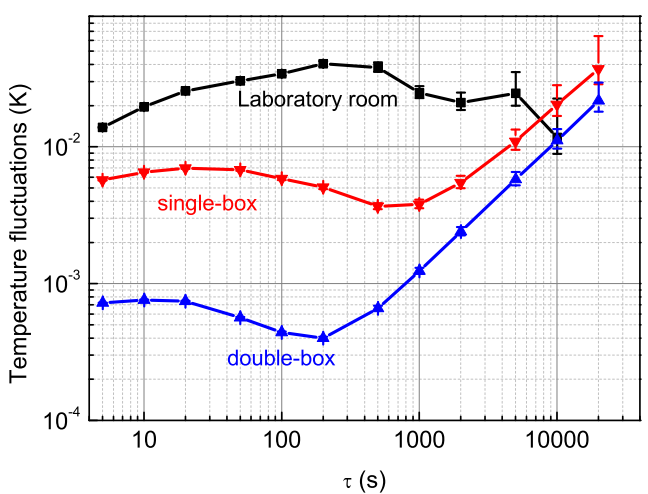

FIG. 4: Allan deviation of temperature fluctuations. All temperatures are measured using high-precision thermistors. The laboratory thermistor is arbitrarily suspended a few centimeters over the second box top surface.

optical setup is not significantly improved with the isolation boxes.

A laser power servo loop dedicated to operate in the pulsed regime and described in Fig. 5 has been implemented at the output of the Michelson system.

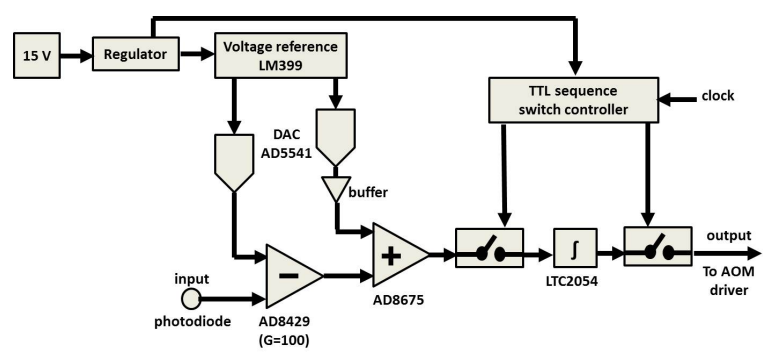

FIG. 5: Simplified schematic of the laser power servo electronics. The photodiode detects an optical light pulse sequence, synchronized and trigged by the pulsed sequence applied onto the AOM. The laser power electronics board reads the photodiode signal when the light is on, generates the error signal by comparison with the reference voltage and applies the correction to the laser power during the pulse. When the light is off, both switches must be opened rapidly with high isolation to prevent any undesired correction.

Here, a small fraction of the laser power is extracted using a beam splitter and monitored by a photodiode to be converted into a dc analog voltage. This output voltage is compared using an ultra-low noise instrumentation amplifier (AD8429, input voltage noise: $1 \mathrm{nV} / \sqrt{\mathrm{Hz}}$, voltage gain 100$)$ to a digitally controlled high-resolution voltage set point extracted from a digital-to-analog converter (DAC AD5541) referenced to a low drift reference voltage (LM399, $0.2 \mathrm{ppm} / \mathrm{K}$ ). The output error signal is summed to an additional digitally-controlled voltage generated by a second DAC referenced to the same LM399 reference voltage. This additional DAC allows in closed-loop oper- ation to tune and control the photodiode output voltage with a $\mu \mathrm{V}$-resolution. The error signal is processed into a PI controller, yielding a correction signal which is applied onto the AOM driving synthesizer RF power. The PI controller is inserted between two high isolation and fast switches (ADG601, $60 \mathrm{~dB}$ isolation, switching time $50 \mathrm{~ns}$ ), switched on and off synchronously with the pulsed Ramsey TTL sequence. The laser power monitoring and the application of the correction signal is performed on each light pulse with a servo bandwidth of about $2 \mathrm{kHz}$. Figure 6 plots the measured relative laser power fluctuations at the input of the CPT cell in free-running and locked regimes. The fractional laser power stability is improved by a factor 1.5 and 40 in the locked regime at $1 \mathrm{~s}$ and $10^{4} \mathrm{~s}$ integration time, respectively.

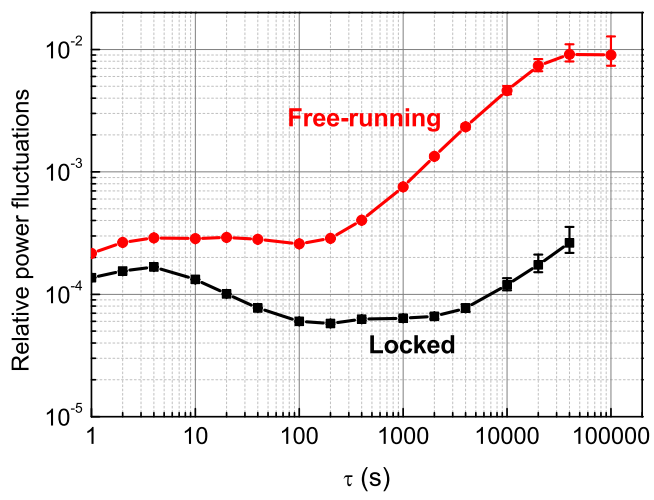

FIG. 6: Allan deviation of relative laser power fluctuations in free-running and locked regimes.

\section{FREQUENCY MEASUREMENTS}

Figure 7 shows a typical Ramsey-CPT fringe detected in the Cs vapor cell for a total input laser power of 800 $\mu \mathrm{W}$ and a free-evolution time $T$ of $2.7 \mathrm{~ms}$. The central fringe linewidth is about $185 \mathrm{~Hz}$. The fringe contrast $C$ defined as the fringe amplitude $(0.35 \mathrm{~V})$ divided by the fringe half-height dc level $(1.835 \mathrm{~V})$ is $19 \%$.

Figure 8 reports the clock frequency shift (offset from $9.192631770 \mathrm{GHz}$ ) versus the laser power in the Ramsey-CPT regime or using the ABR-CPT protocol. In the Ramsey-CPT case, experimental data are wellfitted by a linear function in the studied power range. The light-shift coefficient is in fractional value about $2 \times 10^{-13} / \mu \mathrm{W}$ for $T_{R}=2.7 \mathrm{~ms}$. For optical power lower than $200 \mu \mathrm{W}$, we observed that the frequency shift did not vary linearly with the laser power, as already observed in previous studies $[20,44,65,66]$. Using the ABR-CPT interrogation protocol, experimental data are here fitted by a linear function, yielding a sensitivity in fractional value of about $2.5 \times 10^{-14} / \mu \mathrm{W}$ (with $T_{2}=2.7$ $\mathrm{ms}$ and $T_{1}=5.4 \mathrm{~ms}$ ), a factor 8 times lower than in the 


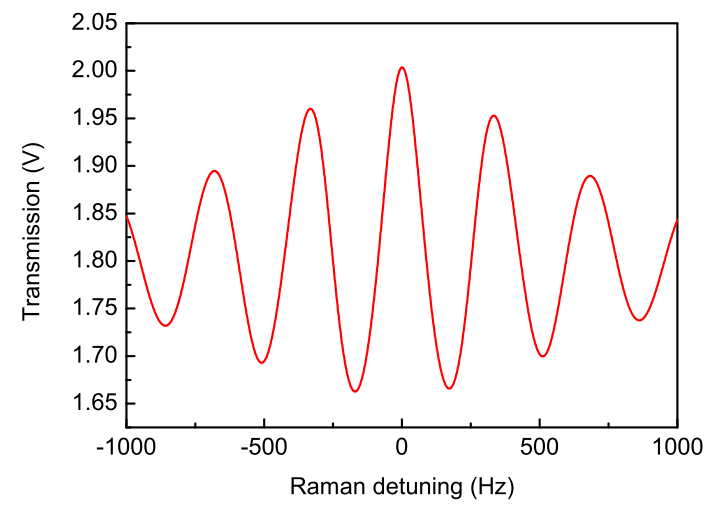

FIG. 7: Typical Ramsey-CPT fringe. The laser power is 800 $\mu \mathrm{W}$ and the free-evolution time $T$ is $2.7 \mathrm{~ms}$.

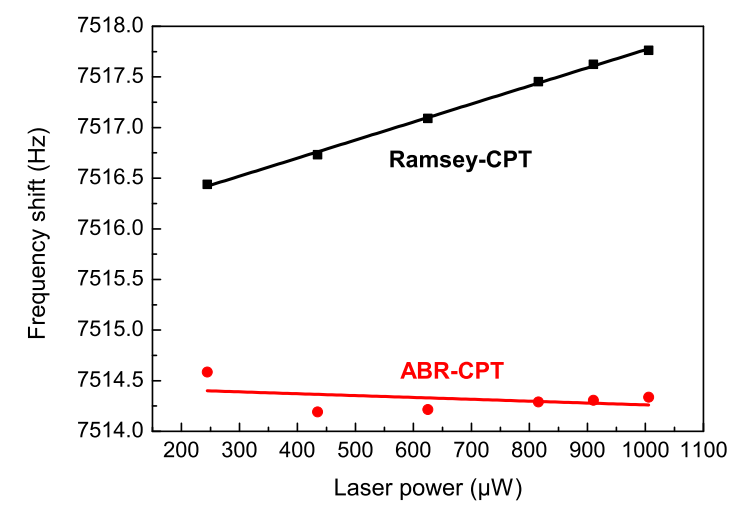

FIG. 8: Clock frequency shift (offset from $9.192631770 \mathrm{GHz}$ ) versus the laser power in the Ramsey-CPT regime or the ABR-CPT protocol. Error bars are included in the size of the dots.

\section{Ramsey-CPT case.}

Figure 9 shows the trajectory of the real system in the phase space $\left(\varphi_{c}, \delta\right)$ after closing both interconnected phase and frequency servo loops of the the ABR-CPT technique. The $\delta=0$ value is defined as the steady state value. The trajectory illustrates the case described in Fig. 1. Here, the staircase shape of the response is not visible because values of $\varphi_{c}$ and $\delta$ are recorded only every 10 iterations. For this reason, the initial phase is not null. A greater number of iterations is also necessary compared to the simplified model of section II. This could be due the warm-up time of the AOM generating the optical pulses, the presence of noise and to the presence of other atomic levels. Here, in a real Cs experiment, optical transitions between 32 levels are involved instead of 3 in our simplified model. The inset of Fig. 9 shows a zoom on the phase - frequency detuning diagram, when both interleaved loops have reached the steady-state. The size of of the final spot region represents typical phase and frequency fluctuations obtained in the steady-state regime, consistent with a short-term fractional frequency stability value of about $3 \times 10^{-13}$ at $1 \mathrm{~s}$.

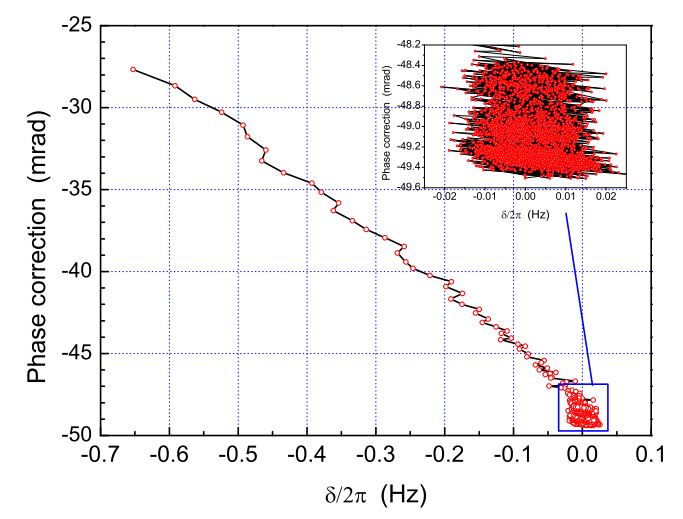

FIG. 9: Example of trajectory of the system in the phase space after closing the frequency and phase loops in ABR technique. The laser power incident in the cell is $800 \mu \mathrm{W}$. Free-evolution times are $2.7 \mathrm{~ms}$ and $5.4 \mathrm{~ms}$. The solid line is a guide for eye. $\delta=0$ is defined as the steady-state value.

Figure 10 reports the Allan deviation of the CPT clock using the Ramsey-CPT mode or the ABR-CPT interrogation protocol. In the Ramsey-CPT case, the short-

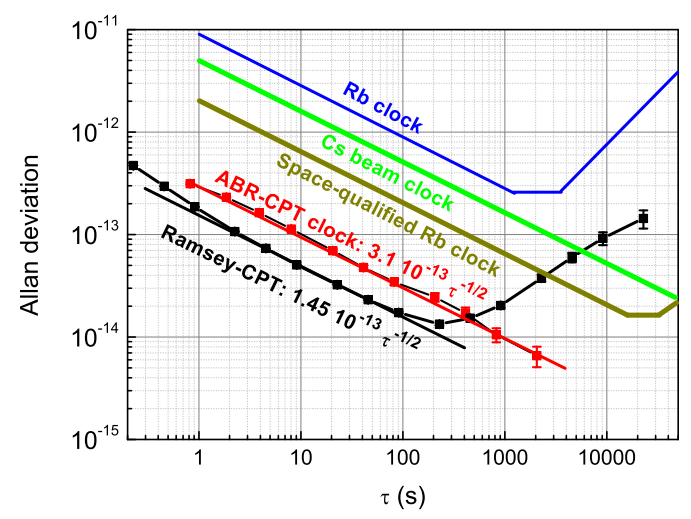

FIG. 10: Allan deviation of the CPT clock using the RamseyCPT or the ABR-CPT interrogation methods. The laser power incident in the cell is $800 \mu \mathrm{W}$. For comparison, performances of a high-performance commercial Cs beam clock, $\mathrm{Rb}$ clock and space-qualified Rb clocks (for GPS systems) [6] are reported.

term fractional frequency stability is improved by a factor 1.6 compared to [13], yielding the level of $1.45 \times$ $10^{-13} \tau^{-1 / 2}$ up to $200 \mathrm{~s}$. This stability improvement is attributed to the reduction of laser power fluctuations thanks to the laser power servo described in section IV. 
Short-term stability performances are here comparable to best Rb cell frequency standards $[14,67]$. The clock Allan deviation at $10^{4} \mathrm{~s}$ averaging time is at the level of $10^{-13}$, a factor 10 better than in Ref. [13]. Here also, the improvement is attributed to improved laser power stabilization. Moreover, at the contrary of the results shown in Ref. [13], the present Allan deviation plot exhibits a well-defined $1 / \sqrt{\tau}$ slope, without any undesired bumps. This minor improvement could be attributed to the enhanced thermal isolation of the experiment.

Using the ABR-CPT protocol, the clock short-term frequency stability is degraded by a factor 2 , yielding $3.1 \times$ $10^{-13}$ at $1 \mathrm{~s}$. This degradation is in correct agreement with the reduction of the Ramsey-CPT fringe signal for $T=5.4 \mathrm{~ms}$, compared to $T=2.7 \mathrm{~ms}$, due to the relaxation of the CPT coherence [13]. Indeed, in this preliminary test, the error signal for the LO frequency correction is extracted from the cycle with long free-evolution time $T_{1}=5.4 \mathrm{~ms}$. Moreover, with the ABR-CPT protocol, the cycle time used for extracting the LO phase correction signal acts as a dead time (loss of information) for the LO frequency servo loop. On the other hand, the Allan deviation of the clock is improved at the level of $6 \times$ $10^{-15}$ at $2000 \mathrm{~s}$ averaging time using the ABR-CPT protocol. These performances represent an improvement by a factor 6.6 compared to the Ramsey-CPT case, in correct agreement with the reduction of the clock frequency sensitivity to laser power, and by a factor 33 compared to our previously published results [13]. Further tests will be performed in the future to evaluate the ABRCPT clock frequency stability on longer time scales, by evaluating the impact of some experimental parameters, optimizing the ABR-CPT sequence or proposing the implementation of variants of the ABR-CPT sequence [68].

\section{CONCLUSIONS}

We have reported a theoretical study and successful experimental implementation of the auto-balanced Ram- sey interrogation protocol to a pulsed Cs cell atomic clock based on coherent population trapping. This original approach allows a compensation of residual uncompensated light shifts induced by the probing laser field, combined with an efficient stabilization of the clock frequency. This method has been found to reduce the sensitivity of the clock frequency to laser power variations. In addition, dedicated electronics have been developed to stabilize the laser power in the pulsed regime and the thermal isolation of the clock experiment has been considered with precaution. The Allan deviation of the ABR-CPT clock is $3.1 \times$ $10^{-13} \tau^{-1 / 2}$, averaging down to the level of $6 \times 10^{-15}$ at $2000 \mathrm{~s}$ integration time. We believe that frequency stability performances of this clock could be improved further in the future by optimizing the ABR-CPT sequence or some of its variants [68] and implementing still-improved electronics. These results reveal exciting perspectives towards the development of low drift CPT-based atomic frequency standards exhibiting numerous potential scientific and industrial applications. The ABR protocol should be also of great interest to be applied in other kinds of compact atomic clocks such as cold atom CPT clocks or pulsed-optically pumped $\mathrm{Rb}$ frequency standards.

\section{Acknowledgments}

This work was led in the frame of a scientific project contract, named PRECISION, funded by LabEX FIRSTTF. G. Coget PhD thesis is funded by Région de FrancheComté. M. Petersen post-doctoral position is supported by LabeX FIRST-TF in the frame of the PRECISION project. The authors thank the platform Oscillator-Imp for the distribution of a reference hydrogen maser signal.

The authors thank P. Abbé (FEMTO-ST) for technical support and help for the implementation of the second temperature insulation box. The authors acknowledge $\mathrm{C}$. Calosso (INRIM) for fruitful and stimulating discussions.
[1] L. S. Cutler, Fifty years of commercial caesium clocks, Met. 42, S90 (2005).

[2] https://www.microsemi.com/product-directory/cesiumfrequency-references/4115-5071a-cesium-primaryfrequency-standard

[3] J. Camparo, The rubidium atomic clock and basic research, Phys. Today 60, 33 (2007).

[4] J. Vanier and C. Mandache, The passive optically pumped Rb standard: the laser approach, Appl. Phys. B 87, 565 (2007).

[5] P. Rochat, F. Droz, P. Mossin, G. Barmaverain, Q. Wang, D. Boving, L. Mattioni, M. Belloni, M. Gioia, U. Schmidt, T. Pike, F. Emma, The Onboard Galileo Rubidium and passive maser, status and performance, Proceedings European Frequency and Time Forum, pp 26-32 (2005).
[6] V. Formichella, J. Camparo and P. Tavella, Influence of the ac-Stark shift on GPS atomic clock timekeeping, Appl. Phys. Lett. 110, 043506 (2017).

[7] A. Godone, F. Levi, C. E. Calosso and S. Micalizio, and A. Godone, High-performing vapor-cell frequency standards, Riv. Nuovo Cimento 38, 3 (2015).

[8] G. Alzetta, A. Gozzini, L. Moi and G. Orriols, An experimental method for the observation of r.f. transitions and laser beat resonances in oriented $\mathrm{Na}$ vapour, Nuovo Cimento B 36, 5 (1976).

[9] E. Arimondo, Coherent population trapping in laser spectroscopy, Prog. Opt., 35, 257 (1996).

[10] J. M. Danet, M. Lours, S. Guérandel and E. De Clercq, Dick Effect in a Pulsed Atomic Clock Using Coherent Population Trapping, IEEE Trans. Ultrason. Ferroelec. Freq. Contr. 61, 4, 567 (2014). 
[11] M. Abdel Hafiz and R. Boudot, A coherent population trapping Cs vapor cell atomic clock based on push-pull optical pumping, J. Appl. Phys. 118, 124903 (2015).

[12] P. Yun, F. Tricot, C. E. Calosso, S. Micalizio, B. Francois, R. Boudot, S. Guérandel, and E. de Clercq, HighPerformance Coherent Population Trapping Clock with Polarization Modulation, Phys. Rev. Applied 7, 014018 (2017).

[13] M. Abdel Hafiz, G. Coget, P. Yun, S. Guérandel, E. de Clercq, and R. Boudot, A high-performance RamanRamsey Cs vapor cell atomic clock, J. Appl. Phys. 121, 104903 (2017)

[14] S. Micalizio, C. E. Calosso, A. Godone and F. Levi, Metrological characterization of the pulsed $\mathrm{Rb}$ clock with optical detection, Metrologia 49, 425-436 (2012).

[15] S. Kang, M. Gharavipour, C. Affolderbach, F. Gruet, and G. Mileti, Demonstration of a high-performance pulsed optically pumped Rb clock based on a compact magnetron-type microwave cavity, J. Appl. Phys. 117, $104510(2015)$.

[16] J. Vanier, M. W. Levine, S. Kendig, D. Janssen, C. Everson and M. J. Delaney, Practical realization of a passive coherent population trapping frequency standard, IEEE Trans. Instr. Meas. 54, 2531 (2005).

[17] N. Castagna, R. Boudot, S. Guérandel, E. de Clercq, N. Dimarcq and A. Clairon, Investigations on Continuous and Pulsed Interrogation for a CPT Atomic Clock, IEEE Trans. Ultrason. Ferroelec. Freq. Contr. 56, (2), 246-253 (2009).

[18] R. Boudot, S. Guérandel, E. de Clercq, N. Dimarcq and A. Clairon, Current Status of a Pulsed CPT Cs Cell Clock, IEEE Trans. Instr. Meas. 58, (4), 1217-1222 (2009).

[19] O. Kozlova, E. de Clercq and S. Guérandel, Temperature and pressure shift of the Cs clock transition in the presence of buffer gases: Ne, $\mathrm{N}_{2}$, Ar, Phys. Rev. A 83, 062714 (2011).

[20] O. Kozlova, J. M. Danet, S. Guérandel, and E. de Clercq, Limitations of Long-Term Stability in a Coherent Population Trapping Cs Clock, IEEE Trans. Instrum. Meas. 63, 1863-1870 (2014).

[21] C. E. Calosso, A. Godone, F. Levi and S. Micalizio, Enhanced Temperature Sensitivity in Vapor-Cell Frequency Standards, IEEE Trans. Ultrason. Ferroelec. Freq. Contr. 59, 12, 2646 (2012).

[22] M. Zhu and L. S. Cutler, Theoretical and experimental study of light shift in a CPT-based Rb vapor cell frequency standard, in Proceedings of the 32nd Annual Precise Time and Time Interval Systems and Applications Meeting, Reston, VA, 2000 (The Institute of Navigation, Manassas, VA, 2000), p. 311.

[23] F.-X. Esnault, E. Blanshan, E.N. Ivanov, R. E. Scholten, J. Kitching, and E. A. Donley, Cold-atom double- $\Lambda$ coherent population trapping clock, Phys. Rev. A 88, 042120 (2013)

[24] P. R. Hemmer, M. S. Shahriar, V. D. Natoli, and S. Ezekiel, AC Stark shifts in a two-zone Raman interaction, J. Opt. Soc. Am. B 6, 1519 (1989).

[25] M. S. Shahriar, P. R. Hemmer, D. P. Katz, A. Lee, and M. G. Prentiss, Dark-state-based three-element vector model for the stimulated Raman interaction, Phys. Rev. A 55, 2272 (1997).

[26] S. Knappe, Emerging topics: MEMS Atomic Clocks, Comp. Microsys. $\underline{3}, 571612$ (2007).
[27] http://www.microsemi.com/products/timingsynchronization-systems/embedded-timingsolutions/components/sa-45s-chip-scale-atomic-clock

[28] V. Shah, V. Gerginov, P. D. D. Schwindt, S. Knappe, L. Hollberg and J. Kitching, Continuous light-shift correction in modulated coherent population trapping clocks, Appl. Phys. Lett. 89, 151124 (2006).

[29] B. H. McGuyer, Y. Y. Jau and W. Happer, Simple method of light-shift suppression in optical pumping systems, Appl. Phys. Lett. 94, 251110 (2009).

[30] Y. Zhang, W. Yang, S. Zhang and J. Zhao, Rubidium chip-scale atomic clock with improved long-term stability through light intensity optimization and compensation for laser frequency detuning, Journ. Soc. Am. B 33, 8, 1756-1763 (2016).

[31] D. Miletic, C. Affolderbach, M. Hasegawa, R. Boudot, C. Gorecki, and G. Mileti, AC Stark-shift in CPT-based Cs miniature atomic clocks, Appl. Phys. B 109, 89-97 (2012).

[32] N.F. Ramsey, A molecular beam resonance method with separated oscillating fields, Phys. Rev. 78, 695 (1950).

[33] J.E. Thomas, P.R. Hemmer, S. Ezekiel, C. C. Leiby, R. H. Picard, and C. R. Willis, Observation of Ramsey Fringes Using a Stimulated, Resonance Raman Transition in a Sodium Atomic Beam, Phys. Rev. Lett. 48, 867 (1982).

[34] P.R. Hemmer, S. Ezekiel, and J. C. C. Leiby, Stabilization of a microwave oscillator using a resonance Raman transition in a sodium beam, Opt. Lett. 8, 440 (1983).

[35] T. Zanon, S. Guérandel, E. de Clercq, D. Holleville, N. Dimarcq and A. Clairon, High Contrast Ramsey Fringes with Coherent-Population-Trapping Pulses in a Double Lambda Atomic System, Phys. Rev. Lett. 94, 193002 (2005).

[36] S. Guérandel, T. Zanon, N. Castagna, F. Dahes, E. De Clercq, N. Dimarcq and A. Clairon, Raman-Ramsey interaction for coherent population trapping Cs clock, IEEE Trans. Instr. Meas. 56, 2, 383 (2007).

[37] G. S. Pati, K. Salit, R. Tripathi and M. S. Shahriar, Demonstration of Raman-Ramsey fringes using time delayed optical pulses in rubidium vapor, Opt. Comm. 281, 4676-4680 (2008).

[38] P. Yun, Y. Zhang, G. Liu, W. Deng, L. You and S. Gu, Multipulse Ramsey-CPT interference fringes for the 87 Rb clock transition, EPL 97, 63004 (2012).

[39] I. Yoshida, N. Hayashi, K. Fujita, S. Taniguchi, Y. Hoshina, and M. Mitsunaga, Line-shape comparison of electromagnetically induced transparency and Raman Ramsey fringes in sodium vapor, Phys. Rev. A 83, 023836 (2013).

[40] J. Yang, Y. Tian, B. Tan, P. Yun and S. Gu, Exploring Ramsey-coherent population trapping atomic clock realized with pulsed microwave modulated laser, J. Appl. Phys. 115, 093109 (2014).

[41] P. Yun, J. M. Danet, D. Holleville, E. de Clercq, S. Guérandel, Constructive polarization modulation for coherent population trapping clock, Appl. Phys. Lett. 105, 231106 (2014).

[42] X. L. Sun, J. W. Wang, P. F. Cheng, C. Xu, L. Zhao and L. J. Wang, Investigation of Ramsey spectroscopy in a lin-par-lin Ramsey coherent population trapping clock with dispersion detection, Opt. Expr. 24, 5, 4532 (2016).

[43] E. Kuchina, E. E. Mikhailov and I. Novikova, Effect of atomic diffusion on the Raman-Ramsey coherent population trapping resonances, J. Opt. Soc. Am. B 33, 4, 
610-614 (2016).

[44] E. Blanshan, S. M. Rochester, E. Donley and J. Kitching, Light shifts in a pulsed cold-atom coherent-populationtrapping clock, Phys. Rev. A 91, 041401(R) (2015).

[45] X. Liu, E. Ivanov, V. I. Yudin, J. Kitching, and E. A. Donley, Low-drift coherent population trapping clock based on laser-cooled atoms and high-coherence excitation fields, Phys. Rev. Appl. 8, 054001 (2017).

[46] X. Liu, V. I. Yudin, A. Taichenachev, J. Kitching, and E. A. Donley, High contrast dark resonances in a cold-atom clock probed with counterpropagating circularly polarized beams, Appl. Phys. Lett. 111, 224102 (2017).

[47] A. Taichenachev, V. Yudin, C. Oates, Z. Barber, N. Lemke, A. Ludlow, U. Sterr, C. Lisdat, and F. Riehle, Compensation of field-induced frequency shifts in Ramsey spectroscopy of optical clock transitions, JETP Lett. 90, $713(2009)$

[48] V. I. Yudin, A. V. Taichenachev, C.W. Oates, Z.W. Barber, N. D. Lemke, A. D. Ludlow, U. Sterr, Ch. Lisdat, and F. Riehle, Hyper-Ramsey spectroscopy of optical clock transitions, Phys. Rev. A 82, 011804(R) (2010).

[49] N. Huntemann, B. Lipphardt, M. Okhapkin, Chr. Tamm, E. Peik, A. V. Taichenachev, and V. I. Yudin, Generalized Ramsey Excitation Scheme with Suppressed Light Shift, Phys. Rev. Lett. 109, 213002 (2012).

[50] R. Hobson, W. Bowden, S. A. King, P. E. G. Baird, I. R. Hill, and P. Gill, Modified hyper-Ramsey methods for the elimination of probe shifts in optical clocks, Phys. Rev. A 93, 010501(R) (2016).

[51] T. Zanon-Willette, E. de Clercq and E. Arimondo, Probe light-shift elimination in generalized hyperRamsey quantum clocks, Phys. Rev. A 93, 042506 (2016).

[52] V. I. Yudin, A. V. Taichenachev, M. V. Okhapkin, S. N. Bagayev, Chr. Tamm, E. Peik, N. Huntemann, T. E. Mehlstaubler, and F. Riehle, Atomic Clocks with Suppressed Blackbody Radiation Shift, Phys. Rev. Lett. 107, 030801 (2011)

[53] V. I. Yudin, A. V. Taichenachev and M. Yu. Basalaev and T. Zanon-Willette, Synthetic frequency protocol for Ramsey spectroscopy of clock transitions, Phys. Rev. A 94, 052505 (2016).

[54] T. Zanon-Willette, R. Lefevre, A. V. Taichenachev, and V. I. Yudin, Universal interrogation protocol with zero probe-field-induced frequency shift for quantum clocks and high-accuracy spectroscopy, Phys. Rev. A 96, 023408 (2017).

[55] C. Sanner, N. Huntemann, R. Lange, C. Tann and E. Peik, Auto-Balanced Ramsey Spectroscopy, Phys. Rev Lett. 120, 053602 (2018).

[56] T. Zanon-Willette, E. de Clercq, and E. Arimondo, Ultrahigh-resolution spectroscopy with atomic or molec- ular dark resonances: Exact steady-state line shapes and asymptotic profiles in the adiabatic pulsed regime, Phys. Rev. A. 84, 062502 (2011).

[57] T. Zanon-Willette, Etudes de transitions atomiques permises et interdites par spectroscopie laser en vue d'une application aux horloges optiques, Habilitation à diriger des Recherches, UPMC, Paris, https://tel.archivesouvertes.fr/tel-00958399v2/document (2014).

[58] Y. Y. Jau, E. Miron, A. B. Post, N. N. Kuzma and W. Happer, Push-pull optical pumping of pure superposition states, Phys. Rev. Lett. 93, 160802 (2004).

[59] X. Liu, J. M. Mérolla, S. Guérandel, C. Gorecki, E. de Clercq and R. Boudot, Coherent population trapping resonances in buffer gas-filled Cs vapor cells with push-pull optical pumping, Phys. Rev. A 87, 013416 (2013).

[60] B. François, C. E. Calosso, J. M. Danet and R. Boudot, A low phase noise microwave frequency synthesis for a high-performance cesium vapor cell atomic clock, Rev. Sci. Instr. 85, 094709 (2014).

[61] M. Abdel Hafiz, G. Coget, E. De Clercq and R. Boudot, Doppler-free spectroscopy on the $\mathrm{Cs}_{\mathrm{D}_{1}}$ line with a dualfrequency laser, Opt. Lett. 41, 13, 2982 (2016).

[62] M. Abdel Hafiz, D. Brazhnikov, G. Coget, A. Taichenachev, V. Yudin, E. de Clercq and R. Boudot, High-contrast sub-Doppler absorption spikes in a hot atomic vapor cell exposed to a dual-frequency laser field, New Journ. Phys. 19, 073028 (2017).

[63] G. A. Pitz, D. E. Wertepny, and G. P. Perram, Pressure broadening and shift of the cesium $\mathrm{D}_{1}$ transition by the noble gases and $\mathrm{N}_{2}, \mathrm{H}_{2}, \mathrm{HD}, \mathrm{D}_{2}, \mathrm{CH}_{4}, \mathrm{C}_{2} \mathrm{H}_{6}, \mathrm{CF}_{4}$, and ${ }^{3}$ He, Phys. Rev. A 80, 062718 (2009).

[64] http://www.t4science.com/documents/iMaser-ClockSpec.pdf (iMaser-ST 3000 specifications).

[65] Y. Yano, W. J. Gao, S. Goka, and M. Kajita, Theoretical and experimental investigation of the light shift in Ramsey coherent population trapping, Phys. Rev. A 90, 013826 (2014).

[66] G. S. Pati, Z. Warren, N. Yu, and M. S. Shahriar, Computational studies of light shift in a RamanRamsey interference-based atomic clock, J. Opt. Soc. Am. B 32, 388-394 (2015).

[67] T. Bandi, C. Affolderbach, C. Stefanucci, F. Merli, A. Skrivervik and G. Mileti, Compact high-performance continuous-wave double-resonance rubidium standard with $1.4 \times 10^{-13} \tau^{-1 / 2}$ stability, IEEE Trans. Ultrason. Ferroelec. Freq. Contr. 61, 11, 1769-1778 (2014).

[68] V. Yudin, A. V. Taichenachev, M. Yu. Basalaev, T. Zanon-Willette, J. W. Pollock, M. Shuker, E. A. Donley, and J. Kitching, Generalized auto-balanced Ramsey spectroscopy of clock transitions, arXiv preprint arXiv:1712.03365 (2017). 